\title{
The Generalized SIC-OEP formalism and the Generalized SIC-Slater approximation (stationary and time-dependent cases).
}

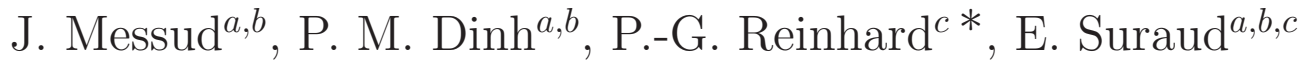 \\ ${ }^{a}$ Université de Toulouse; UPS; \\ Laboratoire de Physique Théorique (IRSAMC); F-31062 Toulouse, France \\ ${ }^{b}$ CNRS; LPT (IRSAMC); F-31062 Toulouse, France \\ ${ }^{c}$ Institut für Theoretische Physik, Universität Erlangen, D-91058 Erlangen, \\ Germany
}

\begin{abstract}
We present a generalized formulation of the Optimized Effective Potential (OEP) approach to the Self Interaction Correction (SIC) problem in Time Dependent (TD) Density Functional Theory (DFT). The formulation relies on the introduction of a double set of single electron orbitals. It allows the derivation of a generalized Slater approximation to the full OEP formulation, which extends the domain of validity of the standard Slater approximation. We discuss both formal aspects and practical applications of the new formalism and give illustrations in cluster and molecules. The new formalism provides a valuable ansatz to more elaborate (and computationally very demanding) full TD OEP and full TD SIC calculations especially in the linear domain.
\end{abstract}

Key words: Time-Dependent Density Functional Theory, Self-Interaction Correction, Irradiation

PACS: 31.15.ee, 31.70.Hq, 34.35.+a, 36.40.Wa, 61.46.Bc

\section{Introduction}

Density Functional Theory (DFT) has evolved to be one of the most powerful theoretical frameworks for the description of complex chemical and physical

\footnotetext{
* Corresponding author

Email-address : Paul-Gerhard.Reinhard@theorie2.physik.uni-erlangen.de
} 
systems. Enormous progress has been made since the seminal works of the sixties by Kohn et al. [1,2]. DFT is now routinely used, especially in systems with a large number of electrons [3 5]. Nevertheless, there remain still several open questions in detail which are in the focus of actual research [6]. The extension to Time-Dependent DFT (TDDFT) has been formally established more recently [7 9]. It is still a developing field, at the side of both formal and practical aspects [10]. Already at the present stage, TDDFT has become one of the few well founded theories for describing the dynamics of complex systems. This is especially true for non equilibrium situations such as clusters and molecules under the influence of intense laser fields [11].

DFT simplifies the involved problem of many-electron correlations in terms of an effective (Hartree-like) one-body description. This is achieved by introducing exchange and correlation effects in an energy functional expressed in terms of the local density of the electrons. The simplest strategy along that line is provided by the Local Density Approximation (LDA) which has proven in many calculations to provide a simple and reliable description of structure and low-amplitude excitations (optical response, one-photon processes) [5]. The analogue in the time-dependent case is the Adiabatic Local Density Approximation (ALDA) which has also been used with great success in dynamical processes involving huge energy deposits and/or large ionization of irradiated clusters and molecules [11].

However, the LDA is plagued by a self-interaction error because the direct Coulomb term and the LDA exchange-correlation potential involve the total density including the particle on which the mean-field acts in the Kohn-Sham equations. While in a full Hartree-Fock treatment, the exchange term exactly cancels the self interaction of the direct term, the approximate treatment of the exchange term in LDA destroys this cancellation. As a consequence, a self-interaction remains and one of the defects is that LDA produces a wrong Coulomb asymptotics [4,12]. The self-interaction thus spoils single-particle properties in particular the Ionization Potential (IP) in finite systems or the band gap in solids [13, 14]. It is also well known that LDA fails in describing the polarizability in chain molecules [15, 16]. In the dynamical case, the selfinteraction error also spoils the description of ionization dynamics, especially close to threshold where IP effects dominate.

There exist ways to correct the self-interaction error while trying to keep the simplicity of the method. An early attempt along that line was proposed by Fermi and Amaldi [17]. The standard way to introduce a Self Interaction Correction (SIC) is based on the more recent proposal of Perdew [12, 18]. Such SIC has been explored since then at various levels of refinement for structure calculations in atomic, molecular, cluster and solid state physics, see e.g. [19 22]. The SIC scheme, however, leads to an orbital dependent meanfield which causes several formal and technical difficulties. A way out is pro- 
vided by using optimized effective potentials (OEP) techniques as introduced in [23, 24], see [25] for a recent review. However, applying OEP to SIC leads to a very involved formalism usually treated with further approximations, as e.g. the Krieger-Li-Iafrate (KLI) approach [26, 27]. But these approximations can severely perturb some crucial physical features of SIC, particularly the trend to produce localized single-particle orbitals [25]. It is thus a key issue to refine such approximate schemes to SIC in order to preserve, as much as possible, original SIC properties, at a lower cost than full OEP. It should be noted that there is nevertheless a further advantage of OEP. It optimizes one local mean-field Hamiltonian for the system. This allows to evaluate unambiguously unoccupied states of the system which, in turn, can have important applications in dynamical processes.

Time-dependent situations call for a time-dependent SIC (TDSIC). Applications of TDSIC have, up to now, mostly been performed in approximate manners, e.g., the linearized treatment of [28], averaged-density SIC [29] based on a generalization of the Amaldi picture [17], or the various versions of timedependent OEP-KLI [30 32]. Only recently, a manageable and exact propagation scheme for TDSIC has been formulated [33, 34] which is applicable in all dynamical ranges. The key to success is to employ two complementing sets of occupied single particle wave functions, one for the mean-field propagation and the other one establishing the necessary localization of the wave functions. The double-set technique has also proven to be extremely useful to formulate efficient approximations to OEP. This was demonstrated for the stationary case in [35, 36] and later on extended to the time domain [37]. The aim of the present paper is to present and discuss in more detail the local approximations to time dependent OEP (TDOEP) based on the double-set technique.

The paper is organized as follows. In section 2, we briefly review the doubleset technique for SIC and TDSIC. In section 3, we introduce the (TD)OEP equations in the light of the double-set representation and develop from that what we call the generalized Slater approximation to OEP. In section 4, we present results for a variety of test cases, static as well as dynamic ones, and compare with results from the lower approximations LDA and ADSIC, and from full TDSIC as a benchmark. Conclusions are summarized in section 5 .

\section{Brief review of the double-set technique for SIC}

In this section, we give a brief outline of SIC and TDSIC in the double-set formulation as introduced in [33, 34. We start from the static case which helps to motivate the double-set technique and proceed to the dynamical case where the use of two sets of wave functions is compulsory. The brief review

of (TD)SIC should serve as a starting point for the derivation of improved 
approximations to OEP (both in stationary and time-dependent cases).

\subsection{Stationary case}

The starting point is the SIC energy functional for electrons :

$$
E_{\mathrm{SIC}}\left[\left\{\psi_{\alpha}\right\}\right]=\sum_{\alpha}\left(\psi_{\alpha}\left|\frac{\mathbf{p}^{2}}{2 m}\right| \psi_{\alpha}\right)+E_{\text {ext }}[\rho]+E_{\mathrm{LDA}}[\rho]-\sum_{\alpha} E_{\mathrm{LDA}}\left[\left|\psi_{\alpha}\right|^{2}\right],
$$

whereby all sums run over occupied states only. Note that we omit the spacetime dependencies, i.e. $\psi_{\alpha}=\psi_{\alpha}(\mathbf{r}, t)$, when it is not misleading. $\rho$ stands for the total electronic density $\rho=\sum_{\alpha} \rho_{\alpha}=\sum_{\alpha}\left|\psi_{\alpha}\right|^{2}$. The first term in $E_{\text {SIC }}$ is the non-interacting kinetic energy; $E_{\text {ext }}[\rho]=\int d \mathbf{r} \rho v_{\text {ext }}$ collects all external one-body fields where $v_{\text {ext }}$ stands for the interaction with the ionic background and any other local possibly time-dependent external field; and $E_{\mathrm{LDA}}[\rho]$ is a standard LDA energy-density functional including the direct term of the electron-electron Coulomb interaction. The last term corresponds to the SIC. We mention in passing that the SIC, and with it all our following development, does also apply to more general functionals as, e.g., the Generalized Gradient Approximation (GGA) [38]. A basic assumption beyond this SIC functional is that the employed single-particle wave functions are ortho-normalized

$$
\left(\psi_{\alpha} \mid \psi_{\beta}\right)=\delta_{\alpha \beta}
$$

The stationary SIC equations are obtained by variation of the SIC energy (11) with the additional constraint on ortho-normalization (2) which is taken into account through the Lagrange multipliers $\lambda_{\alpha \beta}$. This leads to the following mean-field equations [19, 33, 34]

$$
\begin{aligned}
\left.\hat{h}_{\mathrm{SIC}} \mid \psi_{\alpha}\right) & \left.=\sum_{\beta} \lambda_{\alpha \beta} \mid \psi_{\alpha}\right), \\
\hat{h}_{\mathrm{SIC}} & =\hat{h}_{\mathrm{LDA}}-\hat{U}_{\mathrm{SIC}}, \\
\hat{h}_{\mathrm{LDA}} & =\frac{\hat{p}^{2}}{2 m}+U_{\mathrm{LDA}}[\rho], \\
U_{\mathrm{LDA}}[\rho] & =\frac{\delta E_{\mathrm{LDA}}[\rho]}{\delta \rho(\mathbf{r})}, \\
\hat{U}_{\mathrm{SIC}} & \left.=\sum_{\alpha} U_{\mathrm{LDA}}\left[\left|\psi_{\alpha}\right|^{2}\right] \mid \psi_{\alpha}\right)\left(\psi_{\alpha} \mid,\right.
\end{aligned}
$$

combined with what we call the "symmetry condition"

$$
0=\left(\psi_{\beta}\left|U_{\mathrm{LDA}}\left[\left|\psi_{\beta}\right|^{2}\right]-U_{\mathrm{LDA}}\left[\left|\psi_{\alpha}\right|^{2}\right]\right| \psi_{\alpha}\right)
$$


The first term in $\hat{h}_{\mathrm{SIC}}$ is the standard LDA mean field Hamiltonian (30) and the second term stems from the SIC in the energy (1).

Thus far, Eqs. (3) comprise the complete stationary SIC method. The righthand side of Eq. (3a) is unconventional and inconvenient as it does not lead explicitly to single-particle energies. The latter may be defined a posteriori by diagonalizing the matrix of Lagrange multipliers $\lambda_{\alpha \beta}$. We now introduce explicitly a second set of wave functions $\left.\left\{\mid \varphi_{i}\right)\right\}$ which indeed diagonalizes the SIC Hamiltonian,

$$
\left.\left.\hat{h}_{\mathrm{SIC}} \mid \varphi_{i}\right)=\varepsilon_{i} \mid \varphi_{i}\right)
$$

and which is related to the previous set of $\left.\left\{\mid \psi_{\alpha}\right)\right\}$ by a unitary transform amongst occupied states only :

$$
\psi_{\alpha}=\sum_{i} \varphi_{i} u_{i \alpha} \quad, \quad \sum_{i} u_{i \alpha} u_{i \beta}^{*}=\delta_{\alpha \beta}
$$

Both sets lead to the same total density $\rho$ such that the LDA mean-field $U_{\text {LDA }}[\rho]$ remains the same. The new set $\left.\left\{\mid \varphi_{i}\right)\right\}$ represents the energy diagonal states, while the old set $\left.\left\{\mid \psi_{\alpha}\right)\right\}$ remains the decisive ingredient in the symmetry condition (3f). The coefficients $u_{i \alpha}$ of the unitary transformation (5) for given $\varphi_{i}$ are to be determined such that the symmetry condition (3f), involving the $\psi_{\alpha}$, is fulfilled. As the $\varphi_{i}$ orbitals satisfy eigenvalue equations, they are interpreted as single electron orbitals. The set $\psi_{\alpha}$ serves to minimize the SIC energy (11) and to calculate the SIC mean-field $\hat{h}_{\mathrm{SIC}}$.

This completes the double-set representation of stationary SIC. The double-set technique is not compulsory for the stationary case, but enlightening. The two sets play different roles. The energy diagonal states can easily be delocalized and are likely to spread over the whole system, e.g., when considering the valence shell of metallic bonds. The SIC set $\left.\left\{\mid \psi_{\alpha}\right)\right\}$, on the other hand, aims to minimize the SIC energy which is usually achieved by localization of the associated density $\left|\psi_{\alpha}\right|^{2}$ to minimize the Coulomb energy [19, 34, 39].

\subsection{Time-dependent case}

In contrast to the static case, the double-set technique is a necessary ingredient for developing a well-defined and manageable propagation scheme.

To derive the TDSIC equations, we start from the SIC quantum action 


$$
A_{\mathrm{SIC}}=\int_{t_{0}}^{t_{1}} \mathrm{~d} t\left(E_{\mathrm{SIC}}\left[\left\{\psi_{\alpha}\right\}\right](t)-\sum_{\alpha}\left(\psi_{\alpha}(t)\left|\mathrm{i} \hbar \partial_{t}\right| \psi_{\alpha}(t)\right)\right)
$$

The situation with the action for time-dependent variation is, in fact, not so trivial for the derivation of DFT. There can arise problems with causality [40] and boundary conditions [41] for which solutions are discussed in [41,42]. We are dealing here with a local and instantaneous ALDA functional which allows to use the naive action (6). Moreover, concerning the theorems derived from symmetries of the action, it is shown in 42 that, as compensations occur, the stationarity of the naive action (6) leads to the correct final results. We thus perform variation of this action including once again the ortho-normality constraint with Lagrange multipliers $\lambda_{\gamma \beta}$, i.e. we require

$$
\delta\left(A_{\mathrm{SIC}}-\int_{t_{0}}^{t_{1}} d t \sum_{\beta, \gamma}\left(\psi_{\beta}(t) \mid \psi_{\gamma}(t)\right) \lambda_{\gamma \beta}(t)\right)=0
$$

It is to be noted that, to derive the time-dependent OEP formalism, one should use the action (6) in the limit $t_{0} \rightarrow-\infty$. This is necessary to recover in the stationary limit the stationary OEP formalism, as proved in 43 .

The steps of the variation are explained in detail elsewhere [33, 34. We summarize the resulting equations. They again employ the two sets of occupied single-particle wave functions which are connected by a unitary transformation (5). The set $\left.\left\{\mid \varphi_{i}(t)\right)\right\}$, which was the diagonal set in the static case, now turns out to be the "propagating set" obeying the time-dependent mean-field equation

$$
\left.\left(\hat{h}_{\mathrm{SIC}}(t)-i \hbar \partial_{t}\right) \mid \varphi_{i}(t)\right)=0
$$

where $\hat{h}_{\mathrm{SIC}}$ is defined by (3e). The coefficients $u_{i \alpha}$ of the unitary transform (5) for given $\varphi_{i}$ are to be determined such that the "localizing set" $\left.\left\{\mid \psi_{\alpha}(t)\right)\right\}$ satisfies the symmetry condition

$$
u_{i \alpha}(t) \quad: \quad \forall t, 0=\left(\psi_{\beta}(t)\left|U_{\mathrm{LDA}}\left[\left|\psi_{\beta}\right|^{2}\right](t)-U_{\mathrm{LDA}}\left[\left|\psi_{\alpha}\right|^{2}\right](t)\right| \psi_{\alpha}(t)\right)
$$

at any time. The solution scheme for these two coupled equations is obvious. The time-dependent Schrödinger equation (77) for the propagating set is solved for a short time step by standard techniques, e.g., a Taylor expansion of the formal solution $\left.\left.\mid \varphi_{i}\left(t^{\prime}\right)\right)=\exp \left\{-\frac{\mathrm{i}}{\hbar} \int_{t}^{t^{\prime}} \mathrm{d} \tau \hat{h}_{\mathrm{SIC}}(\tau)\right\} \mid \varphi_{i}(t)\right)$. At each time step, the set $\psi_{\alpha}$ is determined by resolving the symmetry condition (3f) [34], which is an instantaneous equation. Then the $\psi_{\alpha}$ serve to construct the new meanfield $\hat{h}_{\text {SIC }}$ for the next time step. 
This TDSIC propagation scheme looks formally straightforward. However, it contains one especially numerical expensive ingredient: The iteration of the symmetry condition (3f) requires to invoke the time-consuming Coulomb solver in each iteration step. Any acceptable approximate solutions are thus welcome. The time-dependent generalized Slater approximation which will be discussed below is a step into this direction.

\section{SIC-OEP and the Generalized SIC-Slater approximation}

\subsection{Stationary formalism}

\subsubsection{SIC-OEP in double-set representation}

The "Optimized Effective Potential" (OEP) formalism is the tool of choice to find the best local approximation to a non-local Hamiltonian. In the present case in which we plan to apply OEP to the SIC problem, we start from the total SIC energy (11) formulated in terms of the (localized) $\psi_{\alpha}$ orbitals and we complement this set by the diagonal orbitals $\varphi_{i}$. The latter are required from the onset to satisfy a local eigenvalue equation

$$
\left(\hat{h}_{\mathrm{LDA}}-U_{\mathrm{SIC}}^{(\text {local })}(\mathbf{r})\right) \varphi_{i}(\mathbf{r})=\epsilon_{i} \varphi_{i}(\mathbf{r})
$$

Locality is imposed by the fact that $U_{\text {SIC }}^{(\text {local })}(\mathbf{r})$ is a function of $\mathbf{r}$ only. The localizing set of $\psi_{\alpha}$ is obtained by the unitary transformation (5) whose coefficients are optimized to minimize the SIC energy (1). It remains to determine the space of occupied single-particle states in terms of the $\varphi_{i}$. The condition (9) shifts the problem to a yet unknown optimizing local potential $U_{\text {SIC }}^{\text {(local) }}$. This potential then becomes the variational degree of freedom instead of the $\varphi_{i}$. The potential $U_{\mathrm{SIC}}^{(\text {local })}(\mathbf{r})$ is thus determined by minimization of the total SIC energy (11)

$$
\frac{\delta E_{\text {SIC }}\left[\left\{\psi_{\alpha}\right\}\right]}{\delta U_{\text {SIC }}^{\text {(local) }}(\mathbf{r})}=0 \quad .
$$

Note that no additional ortho-normality constraint is needed in the variation because it is already guaranteed by solving Eq. (9).

The variation is performed using the chain rule for functional derivatives

$\delta E_{\mathrm{SIC}} / \delta U_{\mathrm{SIC}}^{(\text {local })}=\left(\delta E_{\mathrm{SIC}} / \delta \varphi_{i}^{*}\right)\left(\delta \varphi_{i}^{*} / \delta U_{\text {SIC }}^{\text {(local) }}\right)$ where the first factor represents the usual SIC mean-field and the second factor the wave function response to varied local potential. The detailed derivation is given in [35]. We obtain as a 
final result an integral equation for the $U_{\text {SIC }}^{(\text {local })}$

$$
\sum_{i} \int \mathrm{d} \mathbf{r}^{\prime}\left(U_{\mathrm{SIC}}^{(\text {local })}\left(\mathbf{r}^{\prime}\right)-v_{i}^{*}\left(\mathbf{r}^{\prime}\right)\right) G_{i}\left(\mathbf{r}, \mathbf{r}^{\prime}\right) \varphi_{i}^{*}\left(\mathbf{r}^{\prime}\right) \varphi_{i}(\mathbf{r})=0
$$

where

$$
G_{i}\left(\mathbf{r}, \mathbf{r}^{\prime}\right)=\sum_{j=1}^{+\infty}\left(1-\delta_{i j}\right) \frac{\varphi_{j}^{*}(\mathbf{r}) \varphi_{j}\left(\mathbf{r}^{\prime}\right)}{\epsilon_{j}-\epsilon_{i}}
$$

is the single-particle Green function in the mean-field (9). The driving quantity in the integral equation (11a) is the SIC potential with respect to the $\varphi_{i}$, namely

$$
\begin{aligned}
v_{i}(\mathbf{r}) & =-\frac{1}{\varphi_{i}(\mathbf{r})} \frac{\delta E_{\mathrm{SIC}}\left[\left\{\psi_{\alpha}\right\}\right]}{\delta \varphi_{i}^{*}(\mathbf{r})}+\frac{1}{\varphi_{i}(\mathbf{r})}\left(\mathbf{r}\left|\hat{h}_{\mathrm{LDA}}\right| \varphi_{i}\right) \\
& =\frac{1}{\varphi_{i}(\mathbf{r})} \sum_{\alpha} u_{i \alpha}^{*} U_{\mathrm{LDA}}\left[\left|\psi_{\alpha}\right|^{2}\right](\mathbf{r}) \psi_{\alpha}(\mathbf{r})=\frac{1}{\varphi_{i}(\mathbf{r})}\left(\mathbf{r}\left|\hat{U}_{\mathrm{SIC}}\right| \varphi_{i}\right)
\end{aligned}
$$

where the $\psi_{\alpha}$ are deduced from the $\varphi_{i}$ by the unitary transformation (5) whose coefficients are determined by the symmetry condition (3f). Note that we considered in this variation the diagonal basis states $\varphi_{i}$ and the coefficients $\hat{u}$ of the unitary transformation to the $\psi_{\alpha}$ as independent, i.e. $\delta \hat{u} / \delta \varphi_{i}^{*}(\mathbf{r})=0$ (see also section 4.4 of Ref. 34] where we have shown that the $\varphi_{i}$ and the $u_{i \alpha}$ should be considered as independent in the variation of the energy or the action).

Eq. (11a) defines $U_{\text {SIC }}^{(\text {local })}(\mathbf{r})$ in a rather involved way. Its solution can be disentangled as

$$
\begin{aligned}
U_{\mathrm{SIC}}^{(\text {local })} & =V_{\mathrm{S}}+V_{\mathrm{K}}+V_{\mathrm{C}}, \\
V_{\mathrm{S}} & =\sum_{i} \frac{\left|\varphi_{i}\right|^{2}}{\rho} v_{i}, \\
V_{\mathrm{K}} & =\sum_{i} \frac{\left|\varphi_{i}\right|^{2}}{\rho}\left(\varphi_{i}\left|U_{\mathrm{SIC}}^{(\text {local })}-v_{i}\right| \varphi_{i}\right), \\
V_{\mathrm{C}} & =\frac{1}{2} \sum_{i} \frac{\nabla \cdot\left(p_{i} \nabla\left|\varphi_{i}\right|^{2}\right)}{\rho}, \\
p_{i}(\mathbf{r}) & =\frac{1}{\varphi_{i}^{*}(\mathbf{r})} \int \mathrm{d} \mathbf{r}^{\prime}\left(U_{\mathrm{SIC}}^{\text {(local })}\left(\mathbf{r}^{\prime}\right)-v_{i}^{*}\left(\mathbf{r}^{\prime}\right)\right) \varphi_{i}^{*}\left(\mathbf{r}^{\prime}\right) G_{i}\left(\mathbf{r}, \mathbf{r}^{\prime}\right) .
\end{aligned}
$$

From a practical point of view, this form is not simpler to use than the original form (11a) because the $V_{\mathrm{K}}$ and $V_{\mathrm{C}}$ terms depend on the solution $U_{\text {SIC }}^{\text {(local) }}$. However the separated representation serves as a starting point to develop further approximations. 
Some straightforward manipulations with the unitary transformation (5) allow to rewrite these quantities in terms of the localized wave functions $\psi_{\alpha}$ as

$$
\begin{aligned}
V_{\mathrm{S}} & =\sum_{\alpha} \frac{\left|\psi_{\alpha}\right|^{2}}{\rho} U_{\mathrm{LDA}}\left[\left|\psi_{\alpha}\right|^{2}\right] \\
V_{\mathrm{K}} & =\frac{1}{\rho} \sum_{\alpha, \beta}\left(\sum_{i}\left|\varphi_{i}\right|^{2} u_{i \alpha}^{*} u_{i \beta}\right)\left(\psi_{\beta}\left|U_{\mathrm{SIC}}^{(\text {local })}-U_{\mathrm{LDA}}\left[\left|\psi_{\alpha}\right|^{2}\right]\right| \psi_{\alpha}\right) \\
p_{i}(\mathbf{r}) & =\frac{1}{\varphi_{i}^{*}(\mathbf{r})} \sum_{\alpha} u_{i \alpha} \int \mathrm{d} \mathbf{r}^{\prime}\left(U_{\mathrm{SIC}}^{(\text {local })}\left(\mathbf{r}^{\prime}\right)-U_{\mathrm{LDA}}\left[\left|\psi_{\alpha}\right|^{2}\right]\left(\mathbf{r}^{\prime}\right)\right) \psi_{\alpha}^{*}\left(\mathbf{r}^{\prime}\right) G_{i}\left(\mathbf{r}, \mathbf{r}^{\prime}\right)
\end{aligned}
$$

This expresses SIC-OEP in terms of the double-set representation what we call the "Generalized SIC-OEP" formalism. Thus far it is fully equivalent to the original SIC-OEP equations (111) and as involved to solve. But the doubleset form (13) employs the more localized states $\psi_{\alpha}$ which produces a more forgiving hierarchy of importance for the different terms.

It is to be noted that a very similar development is found in [44], but without addressing the feature of spatial localization of the $\psi_{\alpha}$ when introducing the KLI approximation.

\subsubsection{Generalized SIC-Slater approximation}

In this section, we show that the spatial localization of the $\psi_{\alpha}$ permits to justify a powerful approximation. We define

$$
F_{\alpha}^{(\mathrm{GS})}=\left(U_{\mathrm{LDA}}\left[\left|\psi_{\alpha}\right|^{2}\right]-\sum_{\beta} \frac{\left|\psi_{\beta}\right|^{2}}{\rho} U_{\mathrm{LDA}}\left[\left|\psi_{\beta}\right|^{2}\right]\right) \psi_{\alpha}
$$

In the expected case that the $\psi_{\alpha}$ remain spatially localized, we can assume that at each space point $\mathbf{r}$ one $\psi_{\alpha}$ dominates. This means that

$$
F_{\alpha}^{(\mathrm{GS})} \approx 0
$$

We now take up the "Generalized SIC-OEP" equations (13) and reshuffle them to display the $F_{\alpha}^{(\mathrm{GS})}$ explicitly. The $V_{\mathrm{S}}$ term is dominating compared to the $V_{\mathrm{K}}$ and $V_{\mathrm{C}}$ terms, although those latter terms may be not a priori negligible. Thus we assume approximately

$$
U_{\mathrm{SIC}}^{(\text {local })} \approx V_{\mathrm{S}}=\sum_{\alpha} \frac{\left|\psi_{\alpha}\right|^{2}}{\rho} U_{\mathrm{LDA}}\left[\left|\psi_{\alpha}\right|^{2}\right]
$$


Inserting Eq. (16) into Eqs. (13a) and (13b), we obtain for the two remaining pieces

$$
\begin{aligned}
V_{\mathrm{K}}(\mathbf{r}) & =-\frac{1}{\rho(\mathbf{r})} \sum_{\alpha, \beta}\left(\sum_{i}\left|\varphi_{i}(\mathbf{r})\right|^{2} u_{i \alpha}^{*} u_{i \beta}\right) \int \mathrm{d} \mathbf{r}^{\prime} F_{\alpha}^{(\mathrm{GS})}\left(\mathbf{r}^{\prime}\right) \psi_{\beta}^{*}\left(\mathbf{r}^{\prime}\right) \\
p_{i}(\mathbf{r}) & =-\frac{1}{\varphi_{i}^{*}(\mathbf{r})} \sum_{\alpha} u_{i \alpha} \int \mathrm{d} \mathbf{r}^{\prime} F_{\alpha}^{(G) S *}\left(\mathbf{r}^{\prime}\right) G_{i}\left(\mathbf{r}, \mathbf{r}^{\prime}\right)
\end{aligned}
$$

Using the feature (15) which follows from the localization of the $\psi_{\alpha}$, we obtain

$$
V_{\mathrm{K}} \approx 0 \quad, \quad p_{i} \approx 0 \Rightarrow V_{\mathrm{C}} \approx 0
$$

This justifies a posteriori the assumption (16) and so allows to neglect the more involved contributions $V_{\mathrm{K}}$ and $V_{\mathrm{C}}$ [35]. Note that the double-set technique leaves full freedom for the diagonal orbitals $\varphi_{i}$, whose degree of localization can strongly vary according to the studied system (the $\varphi_{i}$ are, e.g., strongly delocalized in a metal and more localized in covalent binding).

After all, the generalized SIC Slater (GS) approximation to SIC-OEP [35] can be summarized in the three coupled equations

$$
\begin{aligned}
\left.\left(\hat{h}_{\mathrm{LDA}}-\hat{U}_{\mathrm{GS}}\right) \mid \varphi_{i}\right) & \left.=\epsilon_{i} \mid \varphi_{i}\right) \\
\hat{U}_{\mathrm{GS}} & =\sum_{\alpha} \frac{\left|\psi_{\alpha}\right|^{2}}{\rho} \hat{U}_{\mathrm{LDA}}\left[\left|\psi_{\alpha}\right|^{2}\right], \\
0 & =\left(\psi_{\alpha}\left|\hat{U}_{\mathrm{LDA}}\left[\left|\psi_{\alpha}\right|^{2}\right]-\hat{U}_{\mathrm{LDA}}\left[\left|\psi_{\beta}\right|^{2}\right]\right| \psi_{\beta}\right)
\end{aligned}
$$

Eq. (19a) determines the $\varphi_{i}$ for given $\hat{U}_{\mathrm{GS}}$. Eq. (19c) determines the localized states $\psi_{\alpha}$ by finding an appropriate unitary transformation (5). These $\psi_{\alpha}$ are employed in Eq. (19b) to determine the local mean-field $\hat{U}_{\mathrm{GS}}$. The coupled equations can be solved by iteration [35].

\subsubsection{Comment on the traditional SIC-Slater and SIC-KLI approximation}

One can show that the traditional SIC-OEP formalism [26, 27] is obtained by the same reasoning as previously, i.e. imposing that the diagonal orbitals

$\varphi_{i}$ satisfy a local Schrödinger-like equation (9). But having no second set of wave functions at hand, it stops at the stage (12). The traditional SIC-OEP equations [26, 27] are then obtained replacing (11c) by

$$
v_{i}=U_{\mathrm{LDA}}\left[\left|\varphi_{i}\right|^{2}\right]
$$


in the equations (11a) and (12b)-(12e). The traditional SIC-Slater approximation is here formulated as

$$
U_{\mathrm{S}}=\sum_{i} \frac{\left|\varphi_{i}\right|^{2}}{\rho} U_{\mathrm{LDA}}\left[\left|\varphi_{i}\right|^{2}\right]
$$

We discuss its validity in terms of the quantity

$$
F_{i}^{(\mathrm{S})}=\left(U_{\mathrm{LDA}}\left[\left|\varphi_{i}\right|^{2}\right]-\sum_{j} \frac{\left|\varphi_{j}\right|^{2}}{\rho} U_{\mathrm{LDA}}\left[\left|\varphi_{j}\right|^{2}\right]\right) \varphi_{i}
$$

One can shows that the Slater approximation is justified only if

$$
F_{i}^{(\mathrm{S})} \approx 0
$$

i.e. only if the $\varphi_{i}$ orbitals remain spatially localized or very delocalized (close to a Fermi gas). Similar reasoning applies to the traditional KLI approximation. But as extensively discussed previously, the $\varphi_{i}$ have no particular reason to remain localized or to delocalized in the general case. There are many favorable situations as, e.g., a tendency to localized orbitals in organic molecules but also strong delocalization in metallic systems. Thus there is quite a choice of systems where the Slater approximation is found to be applicable. However, there is also a great number of systems where the Slater or KLI approximations fail. This has been numerically shown in 44 for the example of hydrogen chains.

The GS approximation contains with the double-set technique an extra localization step which significantly enhances the range of validity of the Slater approximation. This was shown in terms of several numerical examples in [35, 36 where, e.g., the demanding hydrogen chain was found to be reasonably well described within the GS approximation. A key issue for justifying a Slatertype approximation is that the single electron LDA term $U_{\mathrm{LDA}}\left[\psi_{\alpha}\right]$ is close enough to the density weighted average $\left(\rho_{\alpha} / \rho\right)$ thereof. The approximation obviously works when the $\psi_{\alpha}$ are sufficiently localized, as, for then, around the given point where $\psi_{\alpha}$ is localized, one has $\rho \simeq \rho_{\alpha}$. These strongly localized orbitals correspond to a hydrogen or rare gas bond. There is the other extreme of metallic behavior in which all $\psi_{\alpha}$ extend over the whole system and whose densities resemble each other. This also provides an a priori well working Slater approximation. In between these two extremes range numerous conceivable cases. In particular, they can involve covalent binding which are thus not so well approximated in a simple minded Slater picture. We shall see below that the double set formulation allows to address also such intermediate bindings and performs well in these cases as well. 


\subsection{Time-dependent formalism}

\subsubsection{Time-dependent Generalized SIC-OEP}

We now develop the time-dependent SIC-OEP and "Generalized SIC-Slater" formalisms [37]. Starting point is again the SIC quantum action (6). We impose that the orbitals $\varphi_{i}$ satisfy a time-dependent Schrödinger-like equation with local mean-field potential $U_{\mathrm{SIC}}^{(\text {local })}(\mathbf{r}, t)$, i.e.

$$
\left(h_{\mathrm{LDA}}(\mathbf{r}, t)-U_{\mathrm{SIC}}^{(\mathrm{local})}(\mathbf{r}, t)-i \hbar \partial_{t}\right) \varphi_{i}(\mathbf{r}, t)=0
$$

The optimal $U_{\text {SIC }}^{\text {(local) }}(\mathbf{r}, t)$ is to be determined by variation of the action

$$
\frac{\delta A_{\mathrm{SIC}}}{\delta U_{\text {SIC }}^{(\text {local })}(\mathbf{r}, t)}=0
$$

while the single-particle wave functions $\varphi_{i}$ become potential-dependent quantities. No additional ortho-normalization constraint is needed in this variation because it is already provided by the solution of Eq. (24). The localized set $\psi_{\alpha}$ is again deduced from the $\varphi_{i}$ by the unitary transformation (5) and the transformation coefficients are to be determined by variation of the action. This yields once more the symmetry condition (3fl) to be fulfilled at each instant. It is to be noted that the emerging double set of $\varphi_{i}$ with $\psi_{\alpha}$ is not exactly the same as the solution of TDSIC. Nonetheless we use the same notations for sake of simplicity.

Similarly as in section 3.1.1, the variation (25) is again evaluated with the chain rule for functional derivatives. After a series of formal manipulations, one obtains an integral equation for the optimal local mean-field potential $U_{\mathrm{SIC}}^{(\text {local })}$

$$
\begin{gathered}
\begin{array}{l}
0=\sum_{i} \int_{-\infty}^{t_{1}} \mathrm{~d} t^{\prime} \int d \mathbf{r}^{\prime}\left(U_{\mathrm{SIC}}^{\text {(local })}\left(\mathbf{r}^{\prime}, t^{\prime}\right)-v_{i}^{*}\left(\mathbf{r}^{\prime}, t^{\prime}\right)\right) K_{i}\left(\mathbf{r}, t ; \mathbf{r}^{\prime}, t^{\prime}\right) \varphi_{i}^{*}\left(\mathbf{r}^{\prime}, t^{\prime}\right) \varphi_{i}(\mathbf{r}, t) \\
+ \text { c.c. },
\end{array} \\
K_{i}\left(\mathbf{r}, t ; \mathbf{r}^{\prime}, t^{\prime}\right)=-i \sum_{j=1, j \neq i}^{+\infty} \varphi_{j}^{*}(\mathbf{r}, t) \varphi_{j}\left(\mathbf{r}^{\prime}, t^{\prime}\right) \theta\left(t-t^{\prime}\right), \\
v_{i}(\mathbf{r}, t)=-\frac{1}{\varphi_{i}(\mathbf{r}, t)} \frac{\delta}{\delta \varphi_{i}^{*}(\mathbf{r}, t)} \int_{-\infty}^{t} \mathrm{~d} t^{\prime} E_{\mathrm{SIC}}\left(t^{\prime}\right)+\frac{1}{\varphi_{i}(\mathbf{r}, t)}\left(\mathbf{r}\left|\hat{h}_{\mathrm{LDA}}(t)\right| \varphi_{i}(t)\right) \\
=\frac{1}{\varphi_{i}(\mathbf{r}, t)} \sum_{\alpha} u_{i \alpha}^{*}(t) U_{\mathrm{LDA}}\left[\left|\psi_{\alpha}\right|^{2}\right](\mathbf{r}, t) \psi_{\alpha}(\mathbf{r}, t) .
\end{gathered}
$$

As in the static case, we can decompose $U_{\text {SIC }}^{(\text {local })}$ in terms of separate contributions 


$$
U_{\mathrm{SIC}}^{(\text {local })}=V_{\mathrm{S}}+\Re e\left\{V_{\mathrm{K}}+V_{\mathrm{C}}\right\}-\Im m\left\{V_{\mathrm{TD} 1}+V_{\mathrm{TD} 2}\right\}
$$

where $V_{\mathrm{S}}, V_{\mathrm{K}}, V_{\mathrm{C}}$ are expressed exactly as in (12b)-(12d) but where now the time dependence induces possible complex components we shall analyze further below. However Eq. (12e) defining the $p_{i}$ for Eq. (12d) is to be replaced by

$$
p_{i}(\mathbf{r}, t)=\frac{\int_{-\infty}^{t} \mathrm{~d} t^{\prime} \int \mathrm{d} \mathbf{r}^{\prime}\left(U_{\mathrm{SIC}}^{(\text {local })}\left(\mathbf{r}^{\prime}, t^{\prime}\right)-v_{i}^{*}\left(\mathbf{r}^{\prime}, t^{\prime}\right)\right) \varphi_{i}^{*}\left(\mathbf{r}^{\prime}, t^{\prime}\right) K_{i}\left(\mathbf{r}, t ; \mathbf{r}^{\prime}, t^{\prime}\right)}{\varphi_{i}^{*}(\mathbf{r}, t)}
$$

The potentials $V_{\mathrm{S}}, V_{\mathrm{K}}, V_{\mathrm{C}}$, which also appear in the stationary case, are now complemented by two dynamical contributions

$$
\begin{aligned}
& V_{\mathrm{TD} 1}=\frac{1}{\rho} \sum_{i} \frac{\nabla^{2}\left|\varphi_{i}\right|^{2}}{4} \int_{-\infty}^{t} \mathrm{~d} t^{\prime}\left(\varphi_{i}\left(t^{\prime}\right)\left|v_{i}\left(t^{\prime}\right)\right| \varphi_{i}\left(t^{\prime}\right)\right) \\
& V_{\mathrm{TD} 2}=\frac{1}{\rho} \sum_{i}\left(\left|\varphi_{i}\right|^{2} \frac{\partial p_{i}}{\partial t}+\mathbf{J}_{i} . \nabla p_{i}\right)
\end{aligned}
$$

where $\mathbf{J}_{i}=\frac{1}{2 i}\left(\varphi_{i}^{*} \nabla \varphi_{i}-\varphi_{i} \nabla \varphi_{i}^{*}\right)$ is the current density. Note that the potential $V_{\mathrm{TD} 1}$ contains a time integral, thus memory effects, while the potential $V_{\mathrm{TD} 2}$ involves the time derivative of the $p_{i}$.

The standard way to derive the time-dependent Slater and time-dependent KLI approximations starts from the above separation in terms of the propagating basis $\varphi_{i}$. More robust approximations will be obtained from a formulation in terms of the localizing set $\psi_{\alpha}$. The separation can be remapped to

$$
\begin{aligned}
V_{\mathrm{S}}= & \sum_{\alpha} \frac{\left|\psi_{\alpha}\right|^{2}}{\rho} U_{\mathrm{LDA}}\left[\left|\psi_{\alpha}\right|^{2}\right] \\
V_{\mathrm{K}}= & \frac{1}{\rho} \sum_{\alpha, \beta}\left(\sum_{i}\left|\varphi_{i}\right|^{2} u_{i \alpha}^{*} u_{i \beta}\right)\left(\psi_{\beta}\left|U_{\mathrm{SIC}}^{(\text {local })}-U_{\mathrm{LDA}}\left[\left|\psi_{\alpha}\right|^{2}\right]\right| \psi_{\alpha}\right) \\
p_{i}(\mathbf{r}, t)= & \frac{1}{\varphi_{i}^{*}(\mathbf{r}, t)} \sum_{\alpha} u_{i \alpha}(t) \int_{-\infty}^{t} \mathrm{~d} t^{\prime} \int \mathrm{d} \mathbf{r}^{\prime} \\
& \left(U_{\mathrm{SIC}}^{\text {(local })}\left(\mathbf{r}^{\prime}, t^{\prime}\right)-U_{\mathrm{LDA}}\left[\left|\psi_{\alpha}\right|^{2}\right]\left(\mathbf{r}^{\prime}, t^{\prime}\right)\right) \psi_{\alpha}^{*}\left(\mathbf{r}^{\prime}, t^{\prime}\right) K_{i}\left(\mathbf{r}, t ; \mathbf{r}^{\prime}, t^{\prime}\right) \\
\left(\varphi_{i}\left|v_{i}\right| \varphi_{i}\right)= & \sum_{\alpha, \beta} u_{i \beta} u_{i \alpha}^{*}\left(\psi_{\beta}\left|U_{\mathrm{LDA}}\left[\left|\psi_{\alpha}\right|^{2}\right]\right| \psi_{\alpha}\right) \\
= & \sum_{\alpha, \beta} u_{i \alpha} u_{i \beta}^{*}\left(\psi_{\alpha}\left|U_{\mathrm{LDA}}\left[\left|\psi_{\alpha}\right|^{2}\right]\right| \psi_{\beta}\right)=\left(\varphi_{i}\left|v_{i}\right| \varphi_{i}\right)^{*}
\end{aligned}
$$


The last equation (28d) implies $\Im m\left\{\left(\varphi_{i}\left|v_{i}\right| \varphi_{i}\right)\right\}=0$ which, in turn, yields $\Im m\left\{V_{\mathrm{TD} 1}\right\}=0$. This thus allows to remove this term in the decomposition (27a).

These equations for the optimal local mean-field together with the timedependent mean-field equation (24) and with the symmetry condition (3f) constitute TDSIC-OEP in double set representation. Its solution is by no means simpler than the solution of fully fledged TDSIC. But the equations with explicit separation of the optimal local mean-field provide a good starting point for approximations.

\subsubsection{Time-dependent generalized SIC-Slater approximation}

The reasoning to derive a time-dependent generalized Slater approximation proceeds very similar to the static case (section 3.1.2). We introduce the function $F_{\alpha}^{(\mathrm{GS})}(\mathbf{r}, t)$ defined in (14) and assume the generalized Slater approximation

$$
U_{\mathrm{SIC}}^{(\text {local })} \approx V_{\mathrm{S}}=\sum_{\alpha} \frac{\left|\psi_{\alpha}\right|^{2}}{\rho} U_{\mathrm{LDA}}\left[\left|\psi_{\alpha}\right|^{2}\right]
$$

Inserting into Eqs. (28b) and (28c) yields

$$
\begin{aligned}
& V_{\mathrm{K}}=-\frac{1}{\rho} \sum_{\alpha, \beta}\left(\sum_{i}\left|\varphi_{i}\right|^{2} u_{i \alpha}^{*} u_{i \beta}\right) \int \mathrm{d} \mathbf{r} F_{\alpha}^{(\mathrm{GS})}(\mathbf{r}, t) \psi_{\beta}^{*}(\mathbf{r}, t) \approx 0, \\
& p_{i}(\mathbf{r}, t)=-\frac{1}{\varphi_{i}^{*}(\mathbf{r}, t)} \sum_{\alpha} u_{i \alpha}(t) \int_{-\infty}^{t} \mathrm{~d} t^{\prime} \int \mathrm{d} \mathbf{r}^{\prime} F_{\alpha}^{(\mathrm{GS}) *}\left(\mathbf{r}^{\prime}, t^{\prime}\right) K_{i}\left(\mathbf{r}, t ; \mathbf{r}^{\prime}, t^{\prime}\right) \approx 0, \\
& \Rightarrow \quad V_{\mathrm{TD} 2} \approx 0, \quad V_{\mathrm{C}} \approx 0 .
\end{aligned}
$$

These results are consistent with the approximation (29). Thus the potential (29) is probably a good approximation of the time-dependent SIC-OEP potential for a broad class of problems, as for example when the localization by the symmetry condition (3f) works well. The time-dependent generalized Slater potential (29) has the same form as the stationary GS potential. It does not contain memory effects anymore, which is another consequence of the localization of the $\psi_{\alpha}$.

The emerging scheme is called time-dependent generalized SIC Slater approximation (TDGS). It can be summarized by the coupled equations 


$$
\begin{aligned}
\left.\left(\hat{h}_{\mathrm{LDA}}-U_{\mathrm{GS}}\right) \mid \varphi_{i}\right) & \left.=\mathrm{i} \hbar \partial_{t} \mid \varphi_{i}\right), \\
U_{\mathrm{GS}} & =\sum_{\alpha} \frac{\left|\psi_{\alpha}\right|^{2}}{\rho} U_{\mathrm{LDA}}\left[\left|\psi_{\alpha}\right|^{2}\right], \\
\forall t: 0 & =\left(\psi_{\alpha}\left|U_{\mathrm{LDA}}\left[\left|\psi_{\alpha}\right|^{2}\right]-U_{\mathrm{LDA}}\left[\left|\psi_{\beta}\right|^{2}\right]\right| \psi_{\beta}\right),
\end{aligned}
$$

where the $\psi_{\alpha}$ are obtained from the $\varphi_{i}$ by the unitary transformation (5) which makes the $\psi_{\alpha}$ to satisfy the symmetry condition (30c).

For the same reasons as discussed in the static case, TDGS should represent an improvement to conventional time-dependent SIC-Slater and SIC-KLI approximations to the extent that it opens a larger class of problems for which the approximation is applicable. This will be demonstrated on practical test cases in section 4 .

\subsubsection{Conservation law I: Energy conservation}

Within TDGS, the $\varphi_{i}$ orbitals propagate under the influence of the potential (30b) according to Eq. (30a). The total energy is computed with $E_{\mathrm{SIC}}$ as given in Eq. (11). We remind that variation of $E_{\text {SIC }}$ defines the SIC mean-field $\hat{U}_{\text {SIC }}$ as defined in (3e) :

$$
\begin{aligned}
\frac{\delta}{\delta \varphi_{i}^{*}\left(\mathbf{r}, t^{\prime}\right)} E_{\mathrm{SIC}}\left[\left\{\psi_{\alpha}\right\}\right](t) & =\left(\mathbf{r}\left|\hat{h}_{\mathrm{SIC}}\left(t^{\prime}\right)\right| \varphi_{i}\left(t^{\prime}\right)\right) \delta\left(t-t^{\prime}\right) \\
\hat{h}_{\mathrm{SIC}}(t) & =\hat{h}_{\mathrm{LDA}}(t)-\hat{U}_{\mathrm{SIC}}(t)
\end{aligned}
$$

Energy conservation is an issue for time-independent external fields, i.e. for $\partial_{t} v_{\text {ext }}=0$. The time evolution of the energy thus becomes 


$$
\begin{aligned}
\partial_{t} E_{\mathrm{SIC}}= & \sum_{i} \int \mathrm{d} t^{\prime} \int \mathrm{d} \mathbf{r} \partial_{t} \varphi_{i}^{*}\left(\mathbf{r}, t^{\prime}\right) \frac{\delta}{\delta \varphi_{i}^{*}\left(\mathbf{r}, t^{\prime}\right)} E_{\mathrm{SIC}}\left[\left\{\left|\psi_{\alpha}\right|^{2}\right\}\right](t)+\text { c.c. } \\
= & \sum_{i} \int \mathrm{d} t^{\prime}\left(\partial_{t} \varphi_{i}\left(t^{\prime}\right) \mid \hat{h}_{\mathrm{SIC}}\left(t^{\prime}\right) \varphi_{i}\left(t^{\prime}\right)\right) \delta\left(t-t^{\prime}\right)+\text { c.c. } \\
= & \frac{i}{\hbar} \sum_{i}\left[\left(\hat{h}_{\mathrm{GS}}(t) \varphi_{i}(t) \mid \hat{h}_{\mathrm{SIC}}(t) \varphi_{i}(t)\right)-\left(\hat{h}_{\mathrm{SIC}}(t) \varphi_{i}(t) \mid \hat{h}_{\mathrm{GS}}(t) \varphi_{i}(t)\right)\right] \\
= & \frac{2}{\hbar} \Im m\left\{\sum_{i}\left(\frac{\mathbf{p}^{2}}{2 m} \varphi_{i} \mid \hat{U}_{\mathrm{SIC}} \varphi_{i}\right)-\sum_{i}\left(\frac{\mathbf{p}^{2}}{2 m} \varphi_{i} \mid \hat{U}_{\mathrm{GS}} \varphi_{i}\right)\right\} \\
= & \frac{2}{\hbar} \Im m\left\{-\frac{\hbar^{2}}{2 m} \int \mathrm{d} \mathbf{r} \sum_{\alpha} U_{\mathrm{LDA}}\left[\left|\psi_{\alpha}\right|^{2}\right] \psi_{\alpha} \Delta \psi_{\alpha}^{*}\right. \\
& \left.\quad+\frac{\hbar^{2}}{2 m} \int \mathrm{d} \mathbf{r} \sum_{\beta} \frac{\left|\psi_{\beta}\right|^{2}}{\rho} U_{\mathrm{LDA}}\left[\left|\psi_{\beta}\right|^{2}\right] \sum_{i} \varphi_{i} \Delta \varphi_{i}^{*}\right\} \\
= & \frac{\hbar}{m} \Im m\left\{-\int \mathrm{d} \mathbf{r} \sum_{\alpha} U_{\mathrm{LDA}}\left[\left|\psi_{\alpha}\right|^{2}\right] \psi_{\alpha} \Delta \psi_{\alpha}^{*}\right. \\
& \left.\quad+\int \mathrm{d} \mathbf{r} \sum_{\beta} \frac{\left|\psi_{\beta}\right|^{2}}{\rho} U_{\mathrm{LDA}}\left[\left|\psi_{\beta}\right|^{2}\right] \sum_{\alpha} \psi_{\alpha} \Delta \psi_{\alpha}^{*}\right\}
\end{aligned}
$$

We finally obtain the time variation of the SIC energy (11) within a GS propagation

$$
\partial_{t} E_{\mathrm{SIC}}\left[\left\{\psi_{\alpha}\right\}\right]=-\frac{\hbar}{m} \Im m\left\{\sum_{\alpha} \int \mathrm{d} \mathbf{r} F_{\alpha}^{(\mathrm{GS})}(\mathbf{r}, t) \Delta \psi_{\alpha}^{*}(\mathbf{r}, t)\right\}
$$

where we employed the deviation function $F_{\alpha}^{(\mathrm{GS})}$ from Eq. (14). The relation (32) shows that the energy is not strictly conserved. The quality of energy conservation depends on the quality of the generalized Slater approximation because the deviation is driven by the same function $F_{\alpha}^{(\mathrm{GS})}$ which enters the decision on negligible terms at the end of section 3.2.2.

We compare this result with the traditional time-dependent SIC-Slater propagation. We can show similarly that the time variation of the associated total energy $E_{\mathrm{SIC}}\left[\left\{\varphi_{i}\right\}\right]$, now expressed in terms of the diagonal orbitals $\varphi_{i}$ reads

$$
\partial_{t} E_{\mathrm{SIC}}\left[\left\{\varphi_{i}\right\}\right]=-\frac{\hbar}{m} \Im m\left\{\sum_{i} \int \mathrm{d} \mathbf{r} F_{i}^{(\mathrm{S})}(\mathbf{r}, t) \Delta \varphi_{i}^{*}(\mathbf{r}, t)\right\}
$$

with $F_{i}^{(\mathrm{S})}$ given by Eq. (22). Energy conservation holds for the traditional SIC-Slater scheme if $F_{i}^{(\mathrm{S})} \approx 0$, thus if the physical system as a whole remains homogeneous or very localized. The extra localization for the $\psi_{\alpha}$ in the double-set technique makes it very likely that energy conservation is improved for TDGS. It is known for traditional TDKLI and TD-Slater that energy conservation lasts for a certain time interval after which energy explodes [45]. We 
expect that TDGS has a similar behavior but with a much extended time interval of practical energy conservation, which allows to use it in a wider range of physical situations.

\subsubsection{Conservation law II: Zero Force Theorem}

The Zero Force Theorem (ZFT) states that a time variation of the total electron momentum can be caused only by an "external" potential 45 48, i.e.

$$
\partial_{t}\langle\mathbf{P}\rangle=-\int d \mathbf{r} \rho \nabla v_{\text {ext }}
$$

It stems from the fact that the electron-electron interaction is translational invariant and can not produce a "net" force on the system which, in turn, leads to the ZFT in the form [47]

$$
\forall \tilde{\rho} \quad: \quad \int d \mathbf{r} \tilde{\rho}(\mathbf{r}, t) \nabla U_{\mathrm{mf}}[\tilde{\rho}](\mathbf{r}, t)=0
$$

where $U_{\mathrm{mf}}$ is the local mean-field potential of the considered method. The ZFT holds for the LDA mean-field and the ADSIC one. We now are going to check the ZFT for the TDGS mean-field $U_{\mathrm{GS}}$.

The time evolution of the total momentum is given by

$$
\begin{aligned}
\partial_{t} \sum_{i}\left(\varphi_{i}|\mathbf{p}| \varphi_{i}\right)= & \sum_{i} \int \mathrm{d} \mathbf{r}\left\{\left(\mathbf{r}\left|\hat{v}_{\mathrm{ext}}+\hat{U}_{\mathrm{LDA}}-\hat{U}_{\mathrm{GS}}\right| \varphi_{i}\right)^{*} \nabla \varphi_{i}(\mathbf{r})\right. \\
& \left.+\left(\mathbf{r}\left|\hat{v}_{\mathrm{ext}}+\hat{U}_{\mathrm{LDA}}-\hat{U}_{\mathrm{GS}}\right| \varphi_{i}\right) \nabla \varphi_{i}^{*}(\mathbf{r})\right\} \\
= & \int \mathrm{d} \mathbf{r} v_{\mathrm{ext}} \nabla \rho+\int \mathrm{d} \mathbf{r} U_{\mathrm{LDA}}[\rho] \nabla \rho \\
& -\int \mathrm{d} \mathbf{r} \sum_{\alpha} \frac{\left|\psi_{\alpha}\right|^{2}}{\rho} U_{\mathrm{LDA}}\left[\left|\psi_{\alpha}\right|^{2}\right] \nabla \rho
\end{aligned}
$$

The second term disappears as can be shown by a partial integration and exploiting the ZFT for $U_{\mathrm{LDA}}$. We add $0=\sum_{\alpha} \int \mathrm{d} \mathbf{r} U_{\mathrm{LDA}}\left[\left|\psi_{\alpha}\right|^{2}\right] \nabla\left|\psi_{\alpha}\right|^{2}$ to the third term and reshuffle Eq. (35) to

$$
\partial_{t} \sum_{i}\left(\varphi_{i}|\mathbf{p}| \varphi_{i}\right)=-\int \mathrm{d} \mathbf{r} \rho \nabla v_{\text {ext }}-2 \Re e\left\{\sum_{\alpha} \int \mathrm{d} \mathbf{r} F_{\alpha}^{(\mathrm{GS})}(\mathbf{r}, t) \nabla \psi_{\alpha}^{*}(\mathbf{r}, t)\right\}
$$

where we employ $F_{\alpha}^{(\mathrm{GS})}$ from Eq. (14). Again, we see that the deviation function $F_{\alpha}^{(\mathrm{GS})}$ drives also the term that violates the ZFT. The ZFT is well fulfilled if $F_{\alpha}^{(\mathrm{GS})}$ is small, i.e. if TDGS is valid. In reverse, violation of ZFT and energy conservation is a valuable indicator for the breakdown of TDGS. 
With similar steps we can evaluate the ZFT for the traditional time-dependent Slater approximation and obtain

$$
\partial_{t} \sum_{i}\left(\varphi_{i}|\mathbf{p}| \varphi_{i}\right)=-\int \mathrm{d} \mathbf{r} \rho \nabla v_{\mathrm{ext}}-2 \Re e\left\{\sum_{i} \int \mathrm{d} \mathbf{r} F_{i}^{(\mathrm{S})}(\mathbf{r}, t) \nabla \varphi_{i}^{*}(\mathbf{r}, t)\right\}
$$

where we use the $F_{i}^{(\mathrm{S})}$ from Eq. (22). The ZFT is thus verified within a traditional SIC-Slater propagation only if $F_{i}^{\mathrm{S}} \approx 0$, thus if the physical system remains homogeneous or very localized. We have argued above that the range of $F_{i}^{(\mathrm{S})} \approx 0$ is much smaller than the range of $F_{\alpha}^{(\mathrm{GS})} \approx 0$. This means that TDGS should maintain the ZFT for a longer time span than traditional timedependent SIC-Slater.

\subsection{Alternative localization criteria}

One major effect of the symmetry condition (30c) is that it produces states $\psi_{\alpha}$ which are better localized than the originally given $\varphi_{i}$. This was the particular feature which we employed to motivate TDGS. On the other hand, the symmetry condition is very expensive to solve in practical calculations. Thus it is worth trying to achieve better localization by less demanding equations. There exist many localization criteria [49,50]. After a series of numerical tests with many of these localization criteria, we have found as a best compromise for a localization criterion the spatial variances of the one-body orbitals :

$$
{\overline{\Delta r^{2}}}_{\mathrm{sp}}=\sum_{\alpha}\left[\left(\psi_{\alpha}\left|\mathbf{r}^{2}\right| \psi_{\alpha}\right)-\left(\psi_{\alpha}|\mathbf{r}| \psi_{\alpha}\right)^{2}\right]
$$

where the index "sp" stands for the summed single-particle variances. Minimization of this variance yields the localization equations

$$
0=\left(\psi_{\alpha}\left|\overline{\mathbf{r}_{\alpha}}-\overline{\mathbf{r}_{\beta}}\right| \psi_{\beta}\right) \quad, \quad \overline{\mathbf{r}_{\alpha}}=\left(\psi_{\alpha}|\mathbf{r}| \psi_{\alpha}\right)
$$

which then replaces the symmetry condition (30c) in the TDGS equation. It serves to determine the coefficients of the unitary transformation (5). It is again a non-linear equation which has to be solved iteratively. But the expectation value $\overline{\mathbf{r}_{\alpha}}$ can be computed much faster than the Coulomb field. Thus TDGS with the localization condition (39) is computationally less demanding. We have to see how it performs in practice. 


\section{Numerical results}

\subsection{Brief reminder of the various studied formalisms}

In the following, we will compare the results obtained with (TD)GS and other approaches to those obtained with full (TD)SIC as a benchmark. The corresponding mean-field Hamiltonians are summarized in table1, all being used in one-body Schrödinger-like equations of the form $\left.\left.\hat{h} \mid \varphi_{i}\right)=i \hbar \partial_{t} \mid \varphi_{i}\right)$. Note that for

\begin{tabular}{|l|l|c|}
\hline expression of $\hat{h}$ in $\left.\left.\hat{h} \mid \varphi_{i}\right)=\epsilon_{i} \mid \varphi_{i}\right)$ & method & acronym \\
\hline \hline$\hat{h}_{\mathrm{LDA}}[\rho]$ & LDA & LDA \\
\hline$\hat{h}_{\mathrm{LDA}}[\rho]-\hat{U}_{\mathrm{LDA}}\left[\frac{\rho}{N}\right]$ & Average Density SIC & ADSIC \\
\hline$\hat{h}_{\mathrm{LDA}}[\rho]-\sum_{j} \frac{\left|\varphi_{j}\right|^{2}}{\rho} \hat{U}_{\mathrm{LDA}}\left[\left|\varphi_{j}\right|^{2}\right]$ & Standard SIC Slater & Slat \\
\hline$\hat{h}_{\mathrm{LDA}}[\rho]-\sum_{\alpha} \frac{\left|\psi_{\alpha}\right|^{2}}{\rho} \hat{U}_{\mathrm{LDA}}\left[\left|\psi_{\alpha}\right|^{2}\right]$ & Generalized SIC Slater & $\begin{array}{l}\text { GS(sym) } \\
\text { GS(var) }\end{array}$ \\
$0=\left(\psi_{\beta}\left|U_{\mathrm{LDA}}\left[\left|\psi_{\beta}\right|^{2}\right]-U_{\mathrm{LDA}}\left[\left|\psi_{\alpha}\right|^{2}\right]\right| \psi_{\alpha}\right)$ & & \\
\hline$\left.\hat{h}_{\mathrm{LDA}}[\rho]-\sum_{\alpha} \hat{U}_{\mathrm{LDA}}\left[\left|\psi_{\alpha}\right|^{2}\right] \mid \psi_{\alpha}\right)\left(\psi_{\alpha} \mid\right.$ & full SIC (benchmark) & (TD)SIC \\
$0=\left(\psi_{\beta}\left|U_{\mathrm{LDA}}\left[\left|\psi_{\beta}\right|^{2}\right]-U_{\mathrm{LDA}}\left[\left|\psi_{\alpha}\right|^{2}\right]\right| \psi_{\alpha}\right)$ & & \\
\hline
\end{tabular}

Table 1

The hierarchy of mean-field Hamiltonians, from simple-most LDA (top line) to full TDSIC (bottom line). The right column shows the acronyms used in the figures and discussion.

GS the symmetry condition (3f) should be added for the two last schemes, to define the localized states $\psi_{\alpha}$ required in the corresponding Hamiltonians. As an alternative, we consider the localization criterion (39) derived from minimization of the spatial variance (38), see section 3.3. We abbreviate the scheme using the symmetry condition as "GS(sym)" and the alternative scheme using Eq. (39) as "GS(var)".

The static and dynamical calculations are performed on 3D coordinate-space grid using standard techniques, for details see e.g. [11, 51]. The calculations are restricted to valence electrons. They are the $3 s$ electrons in $\mathrm{Na}$, the $2 s$ and $2 p$ electrons in $\mathrm{C}$, and naturally the $1 s$ electron in $\mathrm{H}$. The coupling of the ionic cores to the valence electrons is described by pseudopotentials. For the $\mathrm{C}$ and $\mathrm{H}$ atoms, we use Goedecker-type pseudopotentials [52] and for $\mathrm{Na}$ atoms the soft local pseudopotentials of [53]. The LDA part employs the exchange-correlation 
energy functional from [54]. ADSIC is performed as explained in [29]. The static solution is done by accelerated gradient iteration [55,56]. Time stepping is done by fourth order Taylor expansion of the exponential propagation operator [57]. The Poisson equation is solved by a fast Fourier technique combined with separate treatment of the long-range terms [58. Polarizabilities are computed from two static calculations where one is performed under the influence of a small static external dipole field.

\subsection{Potential energy surfaces}

The $\mathrm{C}_{2}$ molecule is found to be a critical test case. The electronic structure changes substantially from the spin-saturated, covalently bound dimer ground state to the highly spin polarized asymptotic atomic states. It is demanding for a theory to describe this transition smoothly. Figure 1 shows the Born-

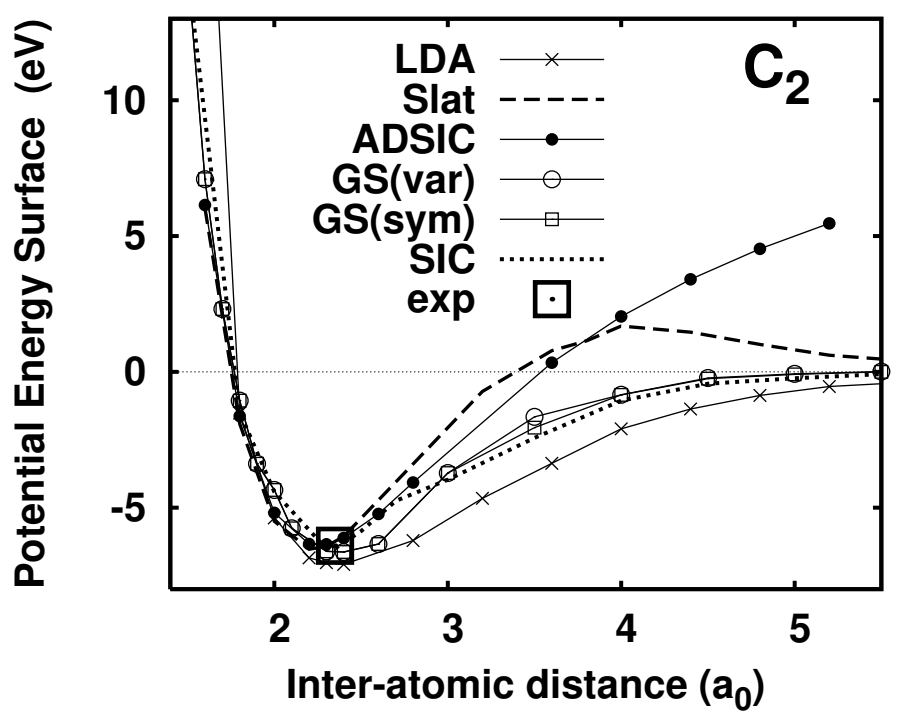

Fig. 1. Potential energy surface of $\mathrm{C}_{2}$ for various $\mathrm{SIC}$ calculations as indicated.

Oppenheimer potential energy surface for the $\mathrm{C}_{2}$ dimer computed with a variety of approaches. Let us first start with the LDA approach which provides a qualitatively good approach with a fair reproduction of both bond length and dissociation energy but which unfortunately underestimates the ground state vibration frequency by about $25 \%$. All SIC corrected methods provide a much better reproduction of the bond length and the dissociation energy of the equilibrium state (less than $5 \%$ of discrepancy with respect to the experimental data). They also improve the value of the vibration frequency : Slater and ADSIC yield an agreement within less than $5 \%$, and full SIC, GS(var) and GS(sym) within typically 10-15\%. Note also that GS(var) deliver results which are almost identical to GS(sym) while being much less expensive numerically. This will also hold in the dynamical regime (see Sec. 4.4). 
However one observes strange behaviors in ADSIC and Slater at intermediate distance with the appearance of a totally unphysical "bump" in the potential energy surface. The effect is not present neither in full SIC nor in GS approximations which thus both provide a correct account of the potential energy surface. The defect observed in Slater and ADSIC has different origin. In Slater, it is probably to be attributed to a conflict between a tendency of the system to create "delocalized" orbitals, to ensure bonding, and a tendency towards "localized" orbitals, to ensure a better account of the SIC. The two set strategy proves here very valuable by resolving the conflict. The ADSIC problem comes from the fact that asymptotically the ADSIC correction should take a form different from that at smaller distance because of the different number of involved electrons ( 4 in each separate $\mathrm{C}$ atom, 8 in the dimer). ADSIC requires compact systems and is generally not suited for describing fragmentation.

\subsection{Polarizabilities}

Polarizabilities are a sensitive test case for density functional approaches [36, 59 65. We will thus discuss this issue here for three sufficiently different systems. As a first example, we consider the carbon molecules $\mathrm{C}_{2}$ and $\mathrm{C}_{4}$.

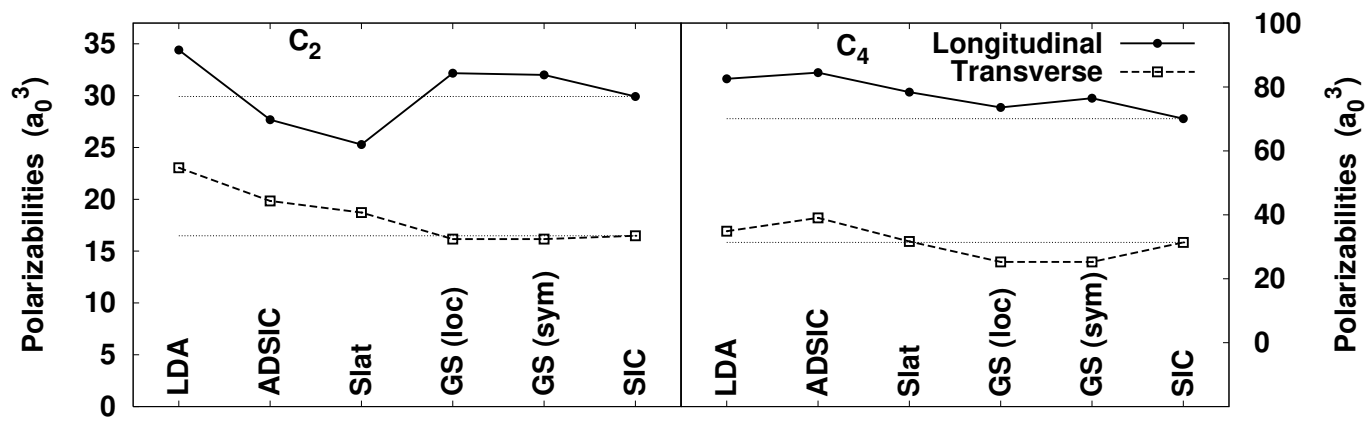

Fig. 2. Transverse and longitudinal polarizabilities of the $\mathrm{C}_{2}$ molecule (left) and the $\mathrm{C}_{4}$ chain (right), calculated in various SIC schemes. Horizontal lines emphasize the SIC benchmark values and ease the comparison with the other results.

Figure 2 shows their polarizabilities for the various approximations. To put the subsequent results on $\mathrm{C}$ molecules into perspective, we recall the computed polarizations for the $\mathrm{C}$ atom: along $z$ axis, $\alpha_{z}=10.40 a_{0}{ }^{3}$ for both SIC and GS, along $x, y$ axes, $\alpha_{x, y}=11.52 a_{0}{ }^{3}$ for GS and $11.76 a_{0}{ }^{3}$ for SIC. Experimental values for the molecular polarizabilities seem not be available. But one can compare with other computed values obtained with much different methods [66, 67] They yield generally comparable values. In [66], the longitudinal polarizability for $\mathrm{C}_{2}$ is $\alpha_{\|}=25 a_{0}{ }^{3}$ for the ab initio methods and $34 a_{0}{ }^{3}$ for LDA/GGA, while the transverse one is $\alpha_{\perp}=25 a_{0}{ }^{3}$ or $100 a_{0}{ }^{3}$ respectively, the latter value being a strange exception. The results for $\mathrm{C}_{4}$ are $\alpha_{\|}=92$ or 
$94 a_{0}{ }^{3}$ and $\alpha_{\perp}=30$ or $32 a_{0}{ }^{3}$. Our results are generally lower for $\alpha_{\perp}$. However, it is to be noted that our calculations differ in the employed functionals and pseudopotentials which both can have a sensitive influence on the results. In view of that, the comparison as a whole looks satisfying.

The main aim of figure 2 is a comparison of methods within the same setup. The $\mathrm{C}_{2}$ dimer shows the larger variance of the results and is obviously more critical than the $\mathrm{C}_{4}$ chain. It is obvious that GS provides the best approximations to full SIC and it is interesting that both versions, GS(sym) and GS(var), perform almost equally well.

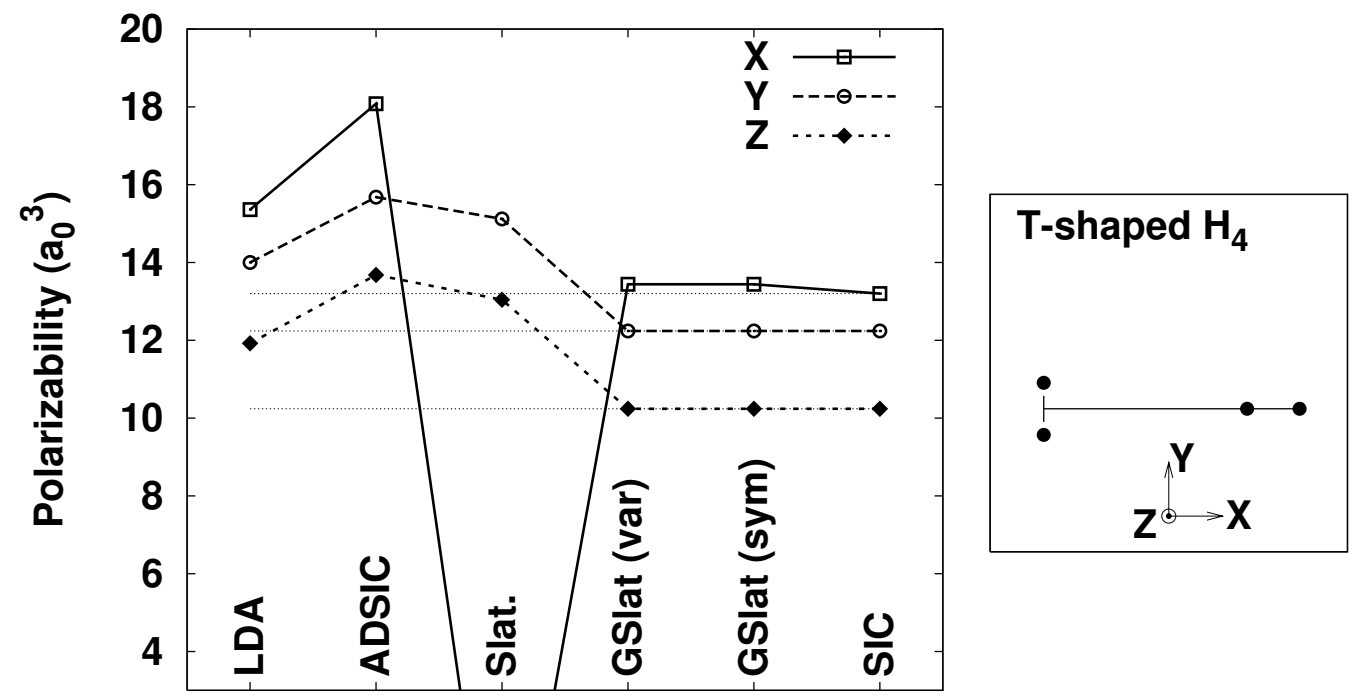

Fig. 3. Polarizabilities, for various SIC schemes as indicated, of the ground state configuration $\mathrm{H}_{4}$ in the T-shaped configuration, displayed in the right panel. Horizontal lines emphasize the SIC benchmark values and ease the comparison with the other results.

The $\mathrm{H}_{4}$ ground state configuration is a "T-shaped" molecule [68] as indicated in the right panel of figure 3 . The $\mathrm{H}_{4}$ ground state configuration consists of two $\mathrm{H}_{2}$ dimers bound with a $\mathrm{H}_{2}-\mathrm{H}_{2}$ center of mass distance of $6.425 \mathrm{a}_{0}$. This is a demanding configuration as it contains two well localized cloud of electrons at each $\mathrm{H}_{2}$ center loosely connected between the centers. Traditional SICSlater and KLI tends to delocalize the wave functions too much. The triaxial spatial configuration provides three different polarizabilities depending on the orientation of the external electric field relative to the molecule. The left panel of figure 3 compares the results for the three polarizabilities. Again we see that both variants of GS come very close to the benchmark (SIC), while LDA, as well as ADSIC, overestimate the polarizabilities, and traditional SIC-Slater is totally off. The overestimation is related to an exaggerated delocalization for LDA and ADSIC. The failure of the traditional Slater approximation indicates a too strong localization of the bonds. GS finds the right compromise. 


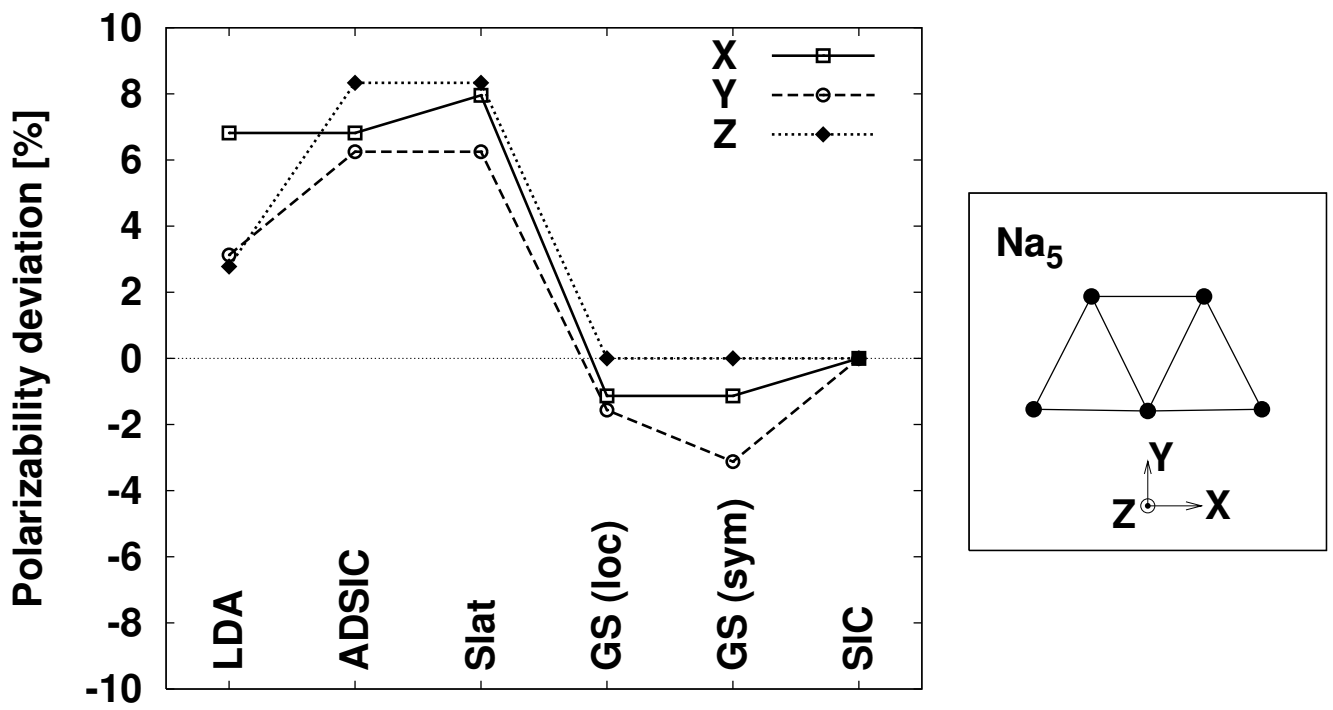

Fig. 4. Polarizabilities, for various SIC schemes as indicated, of $\mathrm{Na}_{5}$, displayed in the right panel. Horizontal lines emphasize the SIC benchmark values and ease the comparison with the other results.

As a final test case for polarizability, we consider the small sodium cluster $\mathrm{Na}_{5}$ representative of simple metallic systems. Being a piece of a metal this cluster should have a delocalized electron cloud. But the rather soft binding of $\mathrm{Na}_{5}$ degrades metalicity and drives towards weak localization. This makes $\mathrm{Na}_{5}$ a particularly critical test case amongst metallic clusters [45. The cluster is planar (see the right panel of figure 4) which corresponds to a triaxial shape and leads to three rather different polarizabilities along the three major axes of the system. In order to display in a better readable way the various results, we have thus chosen to present values relative to the full SIC ones rather than absolute values. The left panel of figure 4 shows the polarizabilities. We obtain much larger absolute values of polarizabilities than in the case of organic systems due to the metallic nature of bonding (delocalization and lower binding). Not surprisingly, all approaches perform rather well, better than in organic systems, as is to be expected for a simple metallic system. Within the lower error bands, we still see differences in the performance with a clear improvement provided by both GS versions.

\subsection{Time-dependent case}

As discussed in sections 3.2 .3 and 3.2.4 Generalized Slater does not exactly fulfill conservation laws and has thus to be used with caution in actual time dependent processes. Still it is interesting to test in dynamical scenarios, especially in the linear domain where it could advantageously replace more complicated approaches. The term linear domain refers to electronic oscillations with 
small amplitudes. In the context of clusters and molecules, it largely refers to the analysis of the optical response which plays a key role in a broad variety of dynamical scenarios, both in the linear and non linear domains [11]. It thus represents a key issue in these systems. TDDFT, in particular in its real time formulation 69, is especially well suited to address such phenomena. For then, the point is simply to excite the system with a sufficiently small energy, whatever its value, so that dynamics sets in and allows a spectral analysis [69]. We shall thus mostly discuss such cases in the following.

\subsection{1 $N a_{5}$}

As a first test case for dynamics, we consider $\mathrm{Na}_{5}$ which was found to be critical probe for studying conservation properties [45] because of its soft and easily polarizable electron cloud. We excite the electronic cloud by applying a boost in the $x$ direction to each wave function. This simulates a very short laser pulse. We compare the case of very small excitation in the linear regime with that of a larger excitation. No absorbing boundary is used here, so that the total energy should be conserved in time.

We first start with the low excitation case presented in Figure 5. The left panel

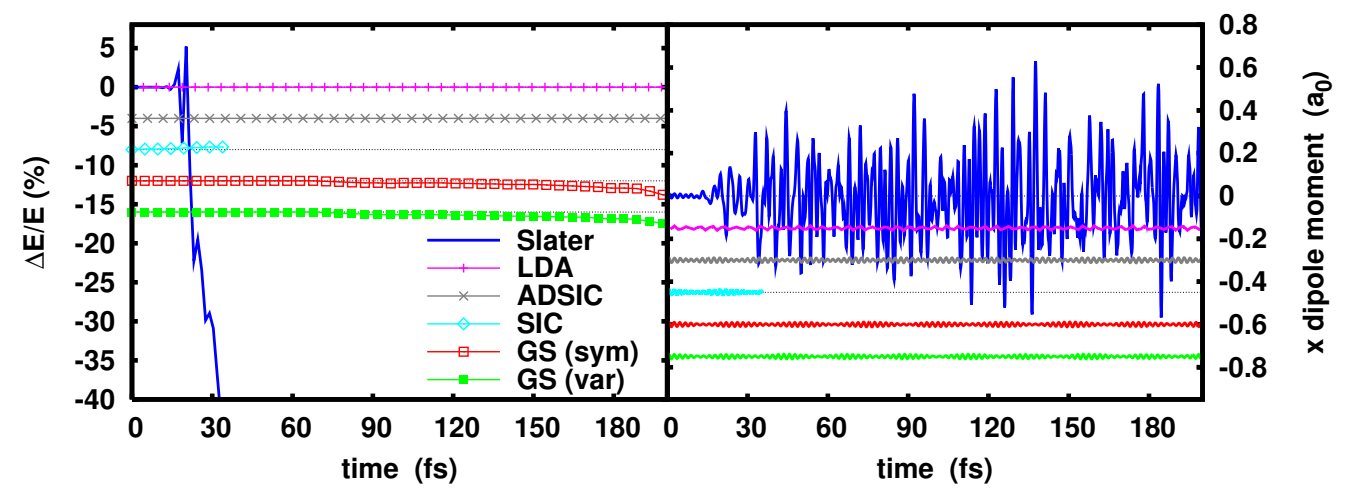

Fig. 5. (Color online) Time evolution of the total energy (left) and the dipole moment in the spatial $x$ direction (right) for $\mathrm{Na}_{5}$ after a boost of the wave functions with momentum $0.001 / \mathrm{a}_{0}$ in the $x$ direction, for various SIC calculations as indicated. For the sake of clarity, some results have been down-shifted by a constant offset.

compares energy conservation. In variational approaches, the total energy is conserved. We thus plot the deviation to this energy conservation, that is $\Delta E / E=[E(t)-E(0)] / E(0)$. As expected, LDA and TDSIC show up as straight lines because these methods are proven to conserve energy. TDGS will finally also develop an energy instability but it stays stable for much longer more than 7 times the standard Slater approximation which diverges after only 30 fs. Moreover, we see that the faster variant GS(var) performs as well as the version employing the involved symmetry condition. The right 
panels of figure 5 show the time evolution of dipole moments. For the sake of clarity, the signals for GS(sym) and GS(var) have been shifted. In agreement with the time evolution of the total energy, in a TDGS calculation, the dipoles do not exhibit any significant evolution, while a standard Slater calculation produces large oscillations.

Figure 6] shows the energy conservation for the same test case $\mathrm{Na}_{5}$ but for a much larger excitation energy 50 times higher, thus in the non-linear regime. We see again that both variants of TDGS provide a longer stability time

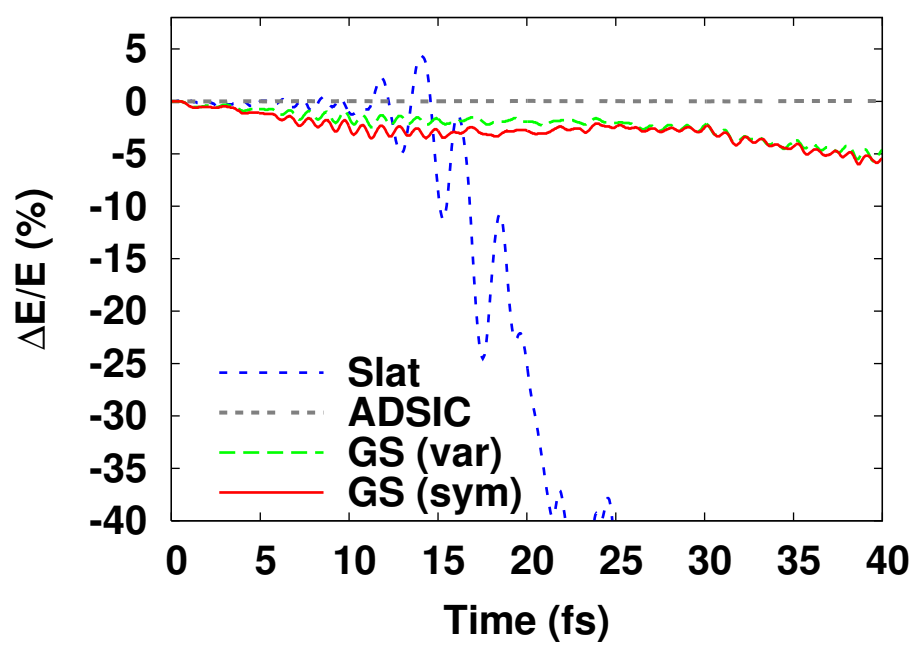

Fig. 6. (Color online) Total energy deviation of $\mathrm{Na}_{5}$ for a boost momentum of $0.05 / \mathrm{a}_{0}$ in the $x$ direction.

than the standard Slater approximation. However, the stability time is much shorter than in the previous case of small excitation. The quality of Slater approximations is degrading with increasing excitation. The applicability has to be checked for each system and dynamical range anew.

\subsubsection{T-shape $\mathrm{H}_{4}$}

We now turn to the case of $\mathrm{H}_{4}$ in the $T$ configuration, as displayed in the top right panel of Figure 7. We excite this cluster the same way, that is with an instantaneous boost. Since GS(var) and GS(sym) turn out to give very close results, we only plot one of these results and denote them as GS without mentioning the criterion used for the calculation of the unitary transform. The findings from the lower two panels are the same as from the previous results. The standard Slater approximation runs rather quickly into violation of energy conservation, while GS stays stable for much longer. The instability in energy shows up at later times in the dipole signal. The upper left panel explores energy conservation for GS at various initial excitation energies. It is apparent that time span at which GS propagates in stable manner depends crucially on 


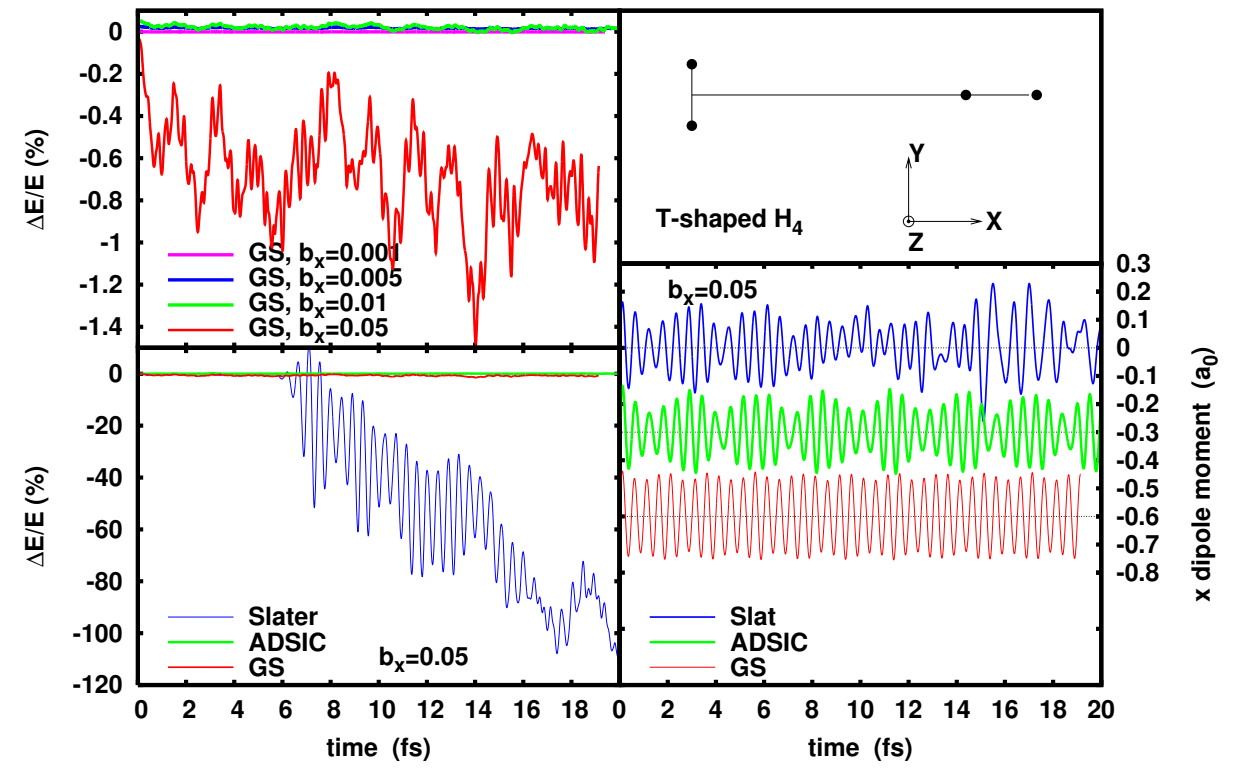

Fig. 7. (Color online) T-shaped $\mathrm{H}_{4}$ (ionic configuration in the top right panel) excited by boosts $b_{x}$ in the $x$ direction as indicated. Left column: total energy deviation as a function of time; bottom right panel: time evolution of the $x$ dipole moment.

the excitation energy of the process. At the present stage of development it can be safely used only in the linear domain.

\section{Conclusion}

We have presented in this paper a generalized formulation of SIC-OEP in the time domain. It relies on the introduction of a double set of electron orbitals. The double-set strategy plays a key role for the time propagation of full SIC but also provides in the OEP context a valuable tool for deriving approximate versions of the full SIC-OEP, in stationary as well as time dependent processes. It allows in particular to introduce the Generalized Slater (GS) approximation which preserves the simplicity of the standard Slater approximation and yet extends its range of validity. While a first set of "in general localized" orbitals serves to fulfill ortho-normality and to construct the approximate Hamiltonian, a second set of "physical" wavefunctions allows a simple time propagation. We have shown that the GS improves over the defects of the simple Slater approximation in particular what concerns conservation laws but still does not allow to fulfill them exactly. We have thus performed various tests on a series of clusters and molecules in order to explore in a practical way the capabilities of GS. We have seen that it performs quite well for static properties and al- 
lows to deal with dynamics in the linear domain. It then provides a simple and transparent alternative to the full TD-OEP or TD-SIC which require substantial numerical effort. The numerical performance of the method still require to be optimized. We have shown that the expensive symmetry condition may be advantageously replaced by the conceptually and computationally simpler "localization" ansatz which opens up possibly new, simplified, approaches to the problem. This strategy nevertheless still requires to be explored in more detail. Work along that line is in progress.

Acknowledgment: This work was supported by a research scholarship from the Alexander-von-Humboldt foundation.

\section{References}

[1] P. Hohenberg, W. Kohn, Phys. Rev. 136 (1964) 864.

[2] W. Kohn, L. J. Sham, Phys. Rev. 140 (1965) 1133.

[3] R. G. Parr, W. Yang, Density-Functional Theory of Atoms and Molecules, Oxford University Press, Oxford, 1989.

[4] R. M. Dreizler, E. K. U. Gross, Density Functional Theory: An Approach to the Quantum Many-Body Problem, Springer-Verlag, Berlin, 1990.

[5] W. Kohn, Rev. Mod. Phys. 71 (1999) 1253-1266.

[6] Http://www.tddft.org/TDDFT2010/program.html.

[7] E. Runge, E. K. U. Gross, Phys. Rev. Lett. 52 (1984) 997.

[8] E. K. U. Gross, W. Kohn, Adv. Quant. Chem. 21 (1990) 255.

[9] M. Marques, E. Gross, Ann. Rev. Phys. Chem. 55 (2004) 427-455.

[10] M. A. Marques, C. A. Ullrich, F. Nogueira, A. Rubio, K. Burke, E. K. Gross, Vol. 706 of Lect. Notes in Phys., Springer, Berlin, 2006.

[11] P.-G. Reinhard, E. Suraud, Wiley, New York, 2003.

[12] J. P. Perdew, A. Zunger, Phys. Rev. B 23 (1981) 5048.

[13] M. S. Hybertsen, S. G. Louie, Phys. Rev. B 34 (1986) 5390.

[14] R. M. Nieminen, Current Opinion in Solid State and Materials Science 4 (1999) 493.

[15] S. J. A. van Gisbergen, P. R. T. Schipper, O. V. Gritsenko, E. J. Baerends, J. G. Snijders, B. Champagne, B. Kirtman, Phys. Rev. Lett. 83 (1999) 694.

[16] S. Kümmel, L. Kronik, J. P. Perdew, Phys. Rev. Lett. 93 (2004) 213002. 
[17] E. Fermi, E. Amaldi, Accad. Ital. Rome 6 (1934) 117.

[18] J. P. Perdew, Chem. Phys. Lett. 64 (1979) 127.

[19] M. R. Pederson, R. A. Heaton, C. C. Lin, J. Chem. Phys. 80 (1984) 1972-1975.

[20] S. Goedecker, C. J. Umrigar, Phys. Rev. A 55 (1997) 1765.

[21] V. Polo, E. Kraka, D. Cremer, Mol. Phys. 100 (2002) 1771.

[22] O. A. Vydrov, G. E. Scuseria, J. Chem. Phys. 121 (2004) 8187.

[23] R. T. Sharp, G. K. Horton, Phys. Rev. 30 (1953) 317.

[24] J. D. Talman, W. F. Shadwick, Phys. Rev. A 14 (1976) 36.

[25] S. Kümmel, L. Kronik, Rev. Mod. Phys. 80 (2008) 3.

[26] J. B. Krieger, Y. Li, G. J. Iafrate, Phys. Rev. A 45 (1992) 101.

[27] J. B. Krieger, Y. Li, G. J. Iafrate, Phys. Rev. A 46 (1992) 5453.

[28] J. M. Pacheco, W. Ekardt, Z. f. Physik D 24 (1992) 65.

[29] C. Legrand, E. Suraud, P.-G. Reinhard, J. Phys. B 35 (2002) 1115.

[30] C. A. Ullrich, U. J. Gossmann, E. K. U. Gross, Phys. Rev. Lett. 74 (1995) 872.

[31] X.-M. Tong, S.-I. Chu, Phys. Rev. A 55 (1997) 3406-3416.

[32] X.-M. Tong, S.-I. Chu, Phys. Rev. A 64 (2001) 013417.

[33] J. Messud, P. M. Dinh, P.-G. Reinhard, E. Suraud, Phys. Rev. Lett. 101 (2008) 096404.

[34] J. Messud, P. M. Dinh, P.-G. Reinhard, E. Suraud, Ann. Phys. (N.Y.) 324 (2009) 955.

[35] J. Messud, P. M. Dinh, P.-G. Reinhard, E. Suraud, Chem. Phys. Lett. 461 (2008) 316.

[36] J. Messud, Z. Wang, P. Dinh, P.-G. Reinhard, E. Suraud, Chem. Phys. Lett. 479 (2009) 300.

[37] J. Messud, P. Dinh, P.-G. Reinhard, E. Suraud, Phys. Rev. A 80 (2009) 044503.

[38] J. P. Perdew, K. Burke, M. Ernzerhof, Phys. Rev. Lett. 77 (1996) 3865.

[39] M. R. Pederson, R. A. Heaton, C. C. Lin, J. Chem. Phys. 82 (1985) 2688.

[40] E. K. U. Gross, C. A. Ullrich, U. J. Gossmann, Vol. 337 of NATO ASI Series B, Plenum Press, New York, 1995, p. 149.

[41] R. van Leeuwen, Phys. Rev. Lett. 80 (1998) 1280.

[42] G. Vignale, Phys. Rev. A 77 (2008) 062511. 
[43] E. Gross, C. Ullrich, U. Grossmann, Density Functional Theory, Plenum Press, New York, 1994.

[44] T. Korzdorfer, S. Kummel, M. Mundt, J. Chem. Phys. 129 (2008) 014110.

[45] M. Mundt, S. Kümmel, R. van Leeuwen, P.-G. Reinhard, Phys. Rev. A 75 (2007) 050501.

[46] M. Levy, J. P. Perdew, Phys. Rev. A 32 (1985) 2010.

[47] G. Vignale, Phys. Rev. Lett. 74 (1995) 3233.

[48] U. von Barth, N. Dahlen, R. van Leeuween, G. Stefanucci, Phys. Rev. B 72 (2005) 235109.

[49] J. M. Foster, S. F. Boys, Rev. Mod. Phys. 32 (1960) 300.

[50] C. Edmiston, K. Ruedenberg, Rev. Mod. Phys. 35 (1963) 457.

[51] F. Calvayrac, P.-G. Reinhard, E. Suraud, C. A. Ullrich, Phys. Rep. 337 (2000) 493.

[52] S. Goedecker, M. Teter, J. Hutter, Phys. Rev. B 54 (1996) 1703.

[53] S. Kümmel, M. Brack, P.-G. Reinhard, Euro. Phys. J. D 9 (1999) 149.

[54] J. P. Perdew, Y. Wang, Phys. Rev. B 45 (1992) 13244.

[55] P.-G. Reinhard, R. Y. Cusson, Nucl. Phys. A 378 (1982) 418.

[56] V. Blum, G. Lauritsch, J. A. Maruhn, P.-G. Reinhard, J. Comp. Phys 100 (1992) 364.

[57] D. Bromley (Ed.), Vol. 3, Plenum, 1985.

[58] G. Lauritsch, P.-G. Reinhard, Int. J. Mod. Phys. C 5 (1994) 65.

[59] B. Champagne, D. H. Mosley, M. Vrako, J.-M. André, Phys. Rev. A 52 (1995) 178.

[60] J. Guan, M. E. Casida, A. M. Koester, D. R. Salahub, Phys. Rev. B 52 (1995) 2184.

[61] A. J. A. van Gisbergen, F. Kootstra, P. R. T. Schipper, O. V. Gritsenko, J. G. Snijders, E. J. Baerends, Phys. Rev. A 57 (1998) 2556.

[62] S. Kümmel, J. Akole, M. Manninen, Phys. Rev. Lett. 84 (2000) 3827.

[63] M. van Faassen, P. L. Boeij, R. van Leeuwen, J. A. Berger, J. G. Snijders, Phys. Rev. Lett. 88 (2002) 186401.

[64] T. Korzdorfer, M. Mundt, S. Kummel, Phys. Rev. Lett. 100 (13) (2008) 133004.

[65] C. D. Pemmaraju, S. Sanvito, K. Burke, Phys. Rev. B. 77 (2008) 121204.

[66] M. Bianchetti, P. F. Buosante, F. Ginelli, H. E. Roman, R. A. Broglia, F. Alasia, Phys. Rep. 357 (2002) 459. 
[67] A. Abdurahman, A. Shukla, G. Seifert, Phys. Rev. B 66 (2002) 155423.

[68] P. Diep, J. K. Johnson, J. Chem. Phys. 112 (2000) 4465.

[69] F. Calvayrac, P.-G. Reinhard, E. Suraud, Ann. Phys. 255 (1997) 125. 